\title{
Efficacy, tolerability and acceptability of oxycodone for cancer-related pain in adults: an updated Cochrane systematic review
}

\author{
Mia Schmidt-Hansen, ${ }^{1}$ Michael I Bennett, ${ }^{2}$ Stephanie Arnold, ${ }^{1}$ \\ Nathan Bromham, ${ }^{1}$ Jennifer S Hilgart ${ }^{3}$
}

- Additional material is published online only. To view please visit the journal online (http://dx.doi.org/10.1136/ bmjspcare-2017-001457)

${ }^{1}$ National Guideline Alliance, Royal College of Obstetricians and Gynaecologists, London, UK ${ }^{2}$ Leeds Institute of Health Sciences, University of Leeds, Leeds, UK

${ }^{3}$ Welsh Institute for Health and Social Care, University of South Wales, Pontypridd, UK

\section{Correspondence to}

Dr Mia Schmidt-Hansen, National Guideline Alliance, Royal College of Obstetricians and Gynaecologists, London NW1 4RG, UK; sapms@cf.ac.uk

Received 26 October 2017 Revised 19 December 2017 Accepted 20 December 2017 Published Online First

13 January 2018

\section{Deck for updates}

To cite: Schmidt-Hansen M, Bennett MI, Arnold S, et al. BMJ Supportive \& Palliative Care 2018;8:117-128.

\section{ABSTRACT \\ Objectives To assess the efficacy, tolerability and acceptability of oxycodone for cancer pain in adults}

Methods We searched CENTRAL, MEDLINE, MEDLINE In-Process, Embase, SCl, Conference Proceedings Citation Index-Science, BIOSIS, PsycINFO and four trials registries to November 2016.

Results We included 23 randomised controlled trials with 2144 patients analysed for efficacy and 2363 for safety. Meta-analyses showed no significant differences between controlledrelease $(C R)$ and immediate-release oxycodone in pain intensity or adverse events but did show significantly better pain relief after treatment with CR morphine compared with CR oxycodone. However, sensitivity analysis did not corroborate this result. Meta-analyses of the adverse events showed a significantly lower risk of hallucinations after treatment with $C R$ oxycodone compared with CR morphine, but no other differences. The remaining studies either compared oxycodone in various formulations or compared oxycodone to different alternative opioids. None found any clear superiority or inferiority of oxycodone in pain relief or adverse events. The quality of this evidence base was limited by the high/unclear risk of bias of the studies and the low event rates for many outcomes.

Conclusions Oxycodone offers similar levels of pain relief and adverse events to other strong opioids. However, hallucinations occurred less with $C R$ oxycodone than with CR morphine, but the quality of this evidence was very low, so this finding should be treated with utmost caution. Our conclusions are consistent with other reviews and suggest that oxycodone can be used first line as an alternative to morphine. However, because it is cheaper, morphine generally remains the first-line opioid of choice.

\section{INTRODUCTION}

Pain from cancer can be caused by direct invasion of a tumour into soft tissue or bone and is often a presenting symptom at the time of diagnosis of cancer. One European survey published in 2009 found that of 5000 people with cancer (including 617 community-based National Health Service (NHS) patients in the UK), 72\% experienced pain $(77 \%$ of UK patients), which was of moderate to severe intensity in $90 \%$ of this group. ${ }^{1}$ This is consistent with a systematic review that demonstrated cancer pain prevalence of up to $75 \%$ in advanced disease and that at least $30 \%$ of people with cancer are undertreated. ${ }^{2}$ Recent research has also shown that less than half of all people with cancer that die are prescribed a strong opioid and that median treatment duration is only 9 weeks before death. ${ }^{3}$ Pain in people with cancer may also be caused by cancer treatments and by comorbid conditions. In this review, we define cancer pain as pain arising as a direct consequence of the cancer and not from other aetiologies.

Oxycodone is a strong opioid analgesic indicated for the treatment of moderate to severe chronic pain, including cancer pain. It is available orally as immediate-release (IR) solution and tablets (for 4-hourly dosing) and as sustained (controlled-release (CR)) release tablets (for 12-hourly dosing). It is also available as a parenteral injection. In some countries, oxycodone is available as a compound with paracetamol (acetaminophen) or ibuprofen.

Oxycodone works primarily as an agonist of mu-opioid receptors in the spinal cord and brain. It has some activity at kappa-opioid receptors (which are also involved in nociception or analgesia) 
though the importance of this mechanism in the overall analgesic effect of oxycodone is unclear. Despite animal studies suggesting differences in pharmacodynamics, these have not been demonstrated in clinical studies to date. Therefore, the shared mechanism of action to other strong opioids (ie, agonist activity at mu-opioid receptors) means that clinical benefits and adverse effects are likely to be similar. However, important differences exist in the pharmacokinetics of strong opioids (eg, morphine undergoes second-phase elimination via glucuronidation, while oxycodone undergoes extensive first-phase metabolism via CYP2D6 and CYP3A4 pathways) so clinical equivalence cannot be inferred. ${ }^{45}$

The WHO published the Method for Cancer Pain Relief (WHO analgesic ladder) in 1986, ${ }^{6}$ which advocates a stepwise approach to analgesia for cancer pain and revolutionised the use of oral opioids. It recommended that morphine be used first line for moderate to severe cancer pain. Observational studies have suggested that this approach results in pain control for $73 \%$ of people ${ }^{7}$ with a mean reduction in pain intensity of $65 \% .^{8}$

Many people with cancer experience moderate to severe pain that requires treatment with strong analgesics. Oxycodone and morphine are examples of strong opioids that are used for the relief of cancer pain. However, strong opioids are not effective for pain in all people, neither are they well tolerated by all people. Guidance by the European Association for Palliative Care on the use of opioids in cancer pain suggests that oxycodone could be used as first-line treatment of moderate to severe cancer pain as an alternative to morphine. ${ }^{9}$ The aim of this review is to assess whether oxycodone is associated with better pain relief (efficacy), tolerability and acceptability than other analgesic options for adults with cancer pain.

This review is an updated Cochrane systematic review on oxycodone for cancer-related pain. ${ }^{10}$

\section{METHODS}

We included randomised controlled trials (RCTs), with parallel group or crossover design, comparing oxycodone (any formulation and any route of administration) with placebo or an active drug (including oxycodone) for cancer background pain in adults (aged $\geq 18$ years). We did not examine studies on breakthrough pain. The studies had to report patient-reported pain intensity/relief with or without adverse events (eg, constipation, nausea, vomiting, drowsiness, confusion, respiratory depression), quality of life (or treatment acceptability as a proxy) and participant preference. If possible, we aimed to distinguish between nociceptive and neuropathic pain, but the data were not presented in a manner that made this possible.

We performed a search of electronic databases (November 2016) and trials registers (December 2016) and checked the bibliographic references of relevant identified studies to find additional trials not identified by the electronic searches and contacted authors of the included studies to ask if they knew of any other relevant studies. We applied no language, date or publication status (published in full, published as abstract and unpublished) restrictions to the search or the study inclusion criteria (see the method section of the online supplementary file for full search strategy).

Two authors independently assessed the study records identified by the search for potential inclusion, extracted data pertaining to study design, participant details (including age, cancer characteristics, previous analgesic medication and setting), interventions (including details about titration) and outcomes and assessed the methodological quality of each of the included studies by using the 'Risk of bias' assessment method outlined in the Cochrane Handbook for Systematic Reviews of Interventions. ${ }^{11}$ We resolved any disagreements by discussion.

For pain intensity, we extracted the means and SD, and we used these to estimate the standardised mean difference (SMD) between the treatments along with the $95 \% \mathrm{CI}$, as the outcome was not measured on the same scale across studies. For adverse events, we extracted event rates to calculate risk ratios (RR) with 95\% CIs as the summary estimates.

The participant was the unit of analysis, but in a number of cases the data reported in the included crossover trials could not otherwise be incorporated into the analyses, so we included them as if the design had been parallel group. This approach gives rise to unit-of-analysis error but is conservative as it results in an underweighting of the data. ${ }^{11}$ However, in order to assess the impact of this strategy, we also performed sensitivity analyses when we included crossover trial data in this manner by excluding the crossover trials from the meta-analyses.

We contacted the authors to request missing data. We limited imputation of missing data to the imputation of missing SDs, either by calculating the SD if enough information was available or by using SDs from similar samples or studies, both according to the methods outlined by Higgins and Green. ${ }^{11}$ We only imputed SDs for pain intensity for Lux et al, ${ }^{12}$ which were not reported for the subgroup of participants with malignant pain, by using the reported SDs for the whole sample of participants with either malignant $(n=31)$ or non-malignant pain $(\mathrm{n}=15)$, and for Yu et $a l^{13}$ for the primary outcome of the study 'mean pain at its worst in the past 24 hours' by using the SDs for the same outcome measured at baseline in the full analysis set. It was not possible to assess the impact of missing data in sensitivity analyses due to the low number of included studies within each comparison. In all cases, we aimed to perform intention-to-treat (ITT) analyses.

We assessed heterogeneity by using the $\mathrm{I}^{2}$ statistic, with $\mathrm{I}^{2}>50 \%$ representing substantial heterogeneity ${ }^{11}$ and had enough data been available, we had 
planned to assess potential sources of heterogeneity through subgroup analyses. Similarly, we had planned to examine the robustness of the meta-analyses by conducting sensitivity analyses using different components of the 'Risk of bias' assessment. Unfortunately, we were unable to perform any such sensitivity analyses due to the low number of studies within each comparison.

We meta-analysed the data extracted from the included studies whenever possible (ie, when the data were reported in a manner that allowed it to be included in the meta-analyses) using Review Manager $5 .{ }^{14}$ We analysed pain intensity using the generic inverse variance method using a fixed-effects model as the $\mathrm{I}^{2}$ statistic was $<50 \%$. We meta-analysed the adverse events data using the Mantel-Haenszel method using a fixed-effects model in all cases; however, as this method is not suitable for crossover trial data, we only included the data from parallel-group trials in these analyses. We used the GRADE system to rank the quality of the evidence using the Grading of Recommendations Assessment, Development and Evaluation (GRADE)profiler Guideline Development Tool software (GRADEpro GDT $2015^{15}$; see the method section of the online supplementary file for further details). This article is a systematic review, so it was not subject to ethical approval.

\section{RESULTS}

The search identified a total of 1121 unique records of which 1014 were excluded based on title/abstract, and the full texts of 107 records were retrieved. Of these 107 records, 23 studies published in 28 articles were included, and 64 records were excluded because they were: not examining the population or comparison of interest (36); systematic (11) or narrative reviews (6); not RCTs/RCT-based analyses (7), letters to the editor (2), duplicates (1) or case reports (1). In addition to these, there were six studies awaiting classification and nine ongoing studies (see the 'Characteristics of ongoing studies' and 'Characteristics of studies awaiting classification' tables, respectively, in the results section of the online supplementary file). A number of the studies identified in the searches compared oxycodone in combination with another drug (eg, naloxone or acetaminophen) against oxycodone alone or placebo. Such studies were not included as they would not answer our primary question, which concerned the effectiveness of oxycodone for cancer pain.

The 23 included studies enrolled/randomised 2648 participants (1347 men, 1109 women; for the remaining 192 participants, gender was not specified) with 2144 of these analysed for efficacy and 2363 for safety. The reported mean/median ages of the participant populations in the studies ranged from 45 years to 68.8 years. Eleven of the studies were crossover trials (published in 16 articles), ${ }^{12}{ }^{16-30}$ and 12 were parallel-group trials (published in 13 articles), ${ }^{13} 31-42$ with 8 of the studies conducted in the USA, ${ }^{16} 1720212930333536392$ in Canada, ${ }^{18} 223$ in Finland, ${ }^{23-25} 3$ in China, ${ }^{13} 40423$ in Italy 3141 and one each in Germany/Poland/Switzerland, ${ }^{12}$ Australia, ${ }^{28}$ Brazil, ${ }^{27}$ the $\mathrm{UK}^{37} 38$ and Japan/ Korea. ${ }^{32}$ The length of the trials ranged from singledose treatment to 1 year, and the studies reported the following comparisons:

- CR oxycodone versus IR oxycodone 2930333639

- CR oxycodone versus extended-release (ER) oxycodone ${ }^{12}$

- CRoxycodoneversusCRmorphine, ${ }^{181923242731343537384142}$ with one of these studies including a further two arms of transdermal (TD) buprenorphine and TD fentayl, ${ }^{31}$ and one of the studies comparing two different brands of slow-release morphine to CR oxycodone $e^{42}$

- CR oxycodone versus CR hydromorphone ${ }^{22}$

- CR oxycodone versus ER hydromorphone ${ }^{13}$

- CR oxycodone versus ER oxymorphone 2021

- CR oxycodone versus ER tapentadol ${ }^{32}$

- CR oxycodone versus TD fentanyl ${ }^{3140}$

- CR oxycodone versus TD buprenorphine ${ }^{31}$

- intravenous oxycodone versus rectal oxycodone $e^{28}$

- intravenous oxycodone followed by IR oxycodone versus intravenous morphine followed by IR morphine ${ }^{25} 26$

- intramuscular (IM) oxycodone vs oral oxycodone ${ }^{16}$

- IM oxycodone versus IM morphine versus IM codeine. ${ }^{17}$

See also Characteristics of included studies in the online supplementary file for further details about the studies.

See figure 1 for summaries of the 'risk of bias' judgements made for the included studies. In the majority of the studies, not enough information was reported to assess whether the methods employed to generate the randomisation sequence and to ensure allocation concealment were adequate. Relatedly, it was also unclear whether the participant selection methods employed had resulted in comparable, balanced groups at study start in the majority of the studies. The risks of performance and detection bias were considered to be low for pain intensity/ relief and adverse events in about half of the studies, unclear in four studies and high in six of the studies that were all described as open label. Overall, the data from only $81 \%$ of the total number of enrolled/ randomised participants were analysed for pain and $89.2 \%$ for adverse events, which indicates that attrition bias was a substantial problem in this data set. Five studies either did not report adverse events or did not report them in a manner so they could be scrutinised for (and potentially included in) an evidence synthesis. ${ }^{1216-19} 27$ One study only reported four adverse events in a transparent manner. ${ }^{22}$ The participants appeared to be adequately titrated in the majority of the studies, ${ }^{12} 13$ 18-27 29-32 35-38 40-42 although this was unclear in four studies, ${ }^{16} 172834$ and not the case in one study. ${ }^{33}$ One study examined titration as its main objective. ${ }^{39}$ For all 11 crossover trials, data were available for all crossover phases. Only four studies undertook ITT analyses 


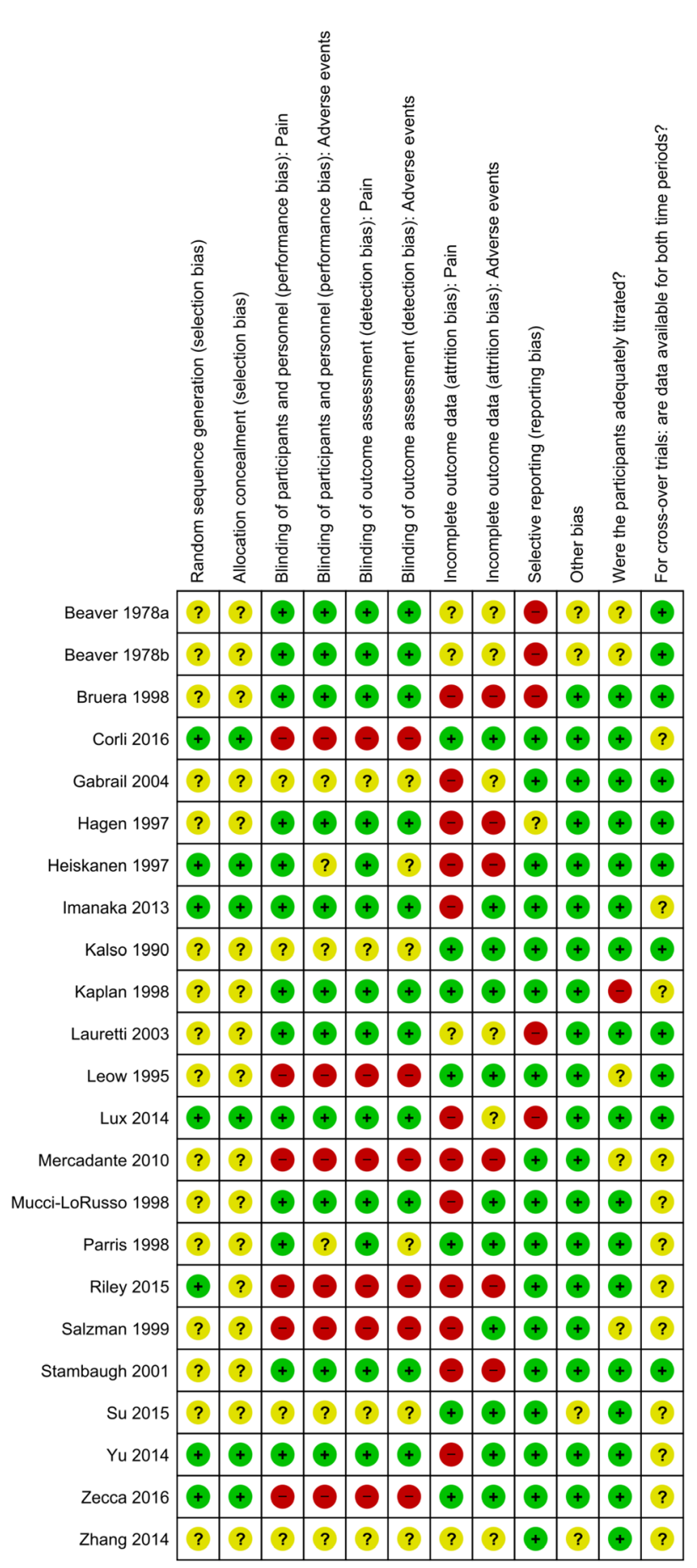

Figure 1 Risk of bias summary: review authors' judgements about each risk of bias item for each included study.

for both efficacy and safety, ${ }^{28-303642}$ with a further three studies performing these analyses for safety only ${ }^{252639}$ or for efficacy only. ${ }^{313738}$ In one study, it was unclear whether ITT analyses had been undertaken. ${ }^{40}$ The remaining studies did not perform ITT for any of the outcomes. The vast majority of the included studies had received commercial funding or had authors who were employees of the drug manufacturers, or both. ${ }^{1213161720-2428-30323335363941}$ Only three studies were considered free from the potential influence of commercial funding, ${ }^{25} 26313738$ with a further five studies having unclear status. ${ }^{18} 1927344042$

\section{Pain}

Figure 2 shows the pain intensity scores for each of the listed treatment groups, subgrouped according to the treatment comparisons.

Pooled analysis showed that there was no statistically significant difference in pain intensity after treatment with either CR or IR oxycodone (SMD $0.1,95 \% \mathrm{CI}-0.06$ to $\left.0.26, \mathrm{I}^{2}=35 \%\right)$. Sensitivity analysis excluding the crossover trial ${ }^{29} 30$ did not change the overall results (SMD $0.12,95 \% \mathrm{CI}-0.06$ to 0.29 ). The study by Salzman et $a l^{39}$ could not be included in the pooled analysis due to the design of the study but found that $\mathrm{CR}$ oxycodone could be used as readily as IR oxycodone for titration to stable pain control with $22 / 24$ and 19/24 participants in the CR and IR groups, respectively, achieving stable pain control within a mean time of 1.6 days $(\mathrm{SE}=0.4)$ and 1.7 days $(\mathrm{SE}=0.6)$, respectively. We judged the quality of evidence for this outcome to be low. We downgraded the quality of evidence by two levels for very serious limitations to study quality due to risk of bias (arising from under-reporting and attrition bias).

Pooled analysis showed that the pain intensity scores were significantly lower after treatment with CR morphine than with CR oxycodone (SMD 0.14, 95\% CI 0.01 to $0.27, \mathrm{I}^{2}=7 \%$ ). Using the $\mathrm{SD}$ of the baseline average pain score of the full sample (200 participants; SD 1.94) from the study by Riley et $a l^{37}{ }^{38}$ to express this SMD as a difference in Brief Pain Inventory scores (0-10 numerical rating scale from no pain to worst pain imaginable) between the treatments gave an estimated difference of 0.27 between the treatments, which was not clinically significant. Moreover, sensitivity analysis excluding the two crossover trials ${ }^{18} 192324$ did not find a significant difference between CR oxycodone and CR morphine (SMD 0.12, 95\% CI -0.02 to $0.26, \mathrm{I}^{2}=24 \%$ ). Two studies could not be included in the pooled analysis due to the design of the study ${ }^{27}$ or because pain intensity was not reported. ${ }^{42}$ Lauretti et al conducted a two-phase (each lasting 14 days) crossover study to examine IR morphine consumption during treatment with $\mathrm{CR}$ oxycodone and CR morphine, keeping the ratio of CR oxycodone and CR morphine constant $(1: 1.8){ }^{27}$ IR morphine was used as rescue medication, and the participants were allowed to take as much as necessary to keep visual analogue scale pain score $<4$. The participants consumed 38\% more IR morphine during treatment with CR morphine than with CR oxycodone. Lauretti et al concluded that the results indicated that CR oxycodone combined with IR morphine was associated with superior analgesia and lower, or similar, rates of adverse events (see 'Adverse events' below) than a combination of CR and IR morphine. ${ }^{27}$ Zhang et al conducted a three-arm parallel-group trial of unknown duration comparing CR oxycodone to CR morphine and CR MS Contin and found that pain relief rates (ie, participants 


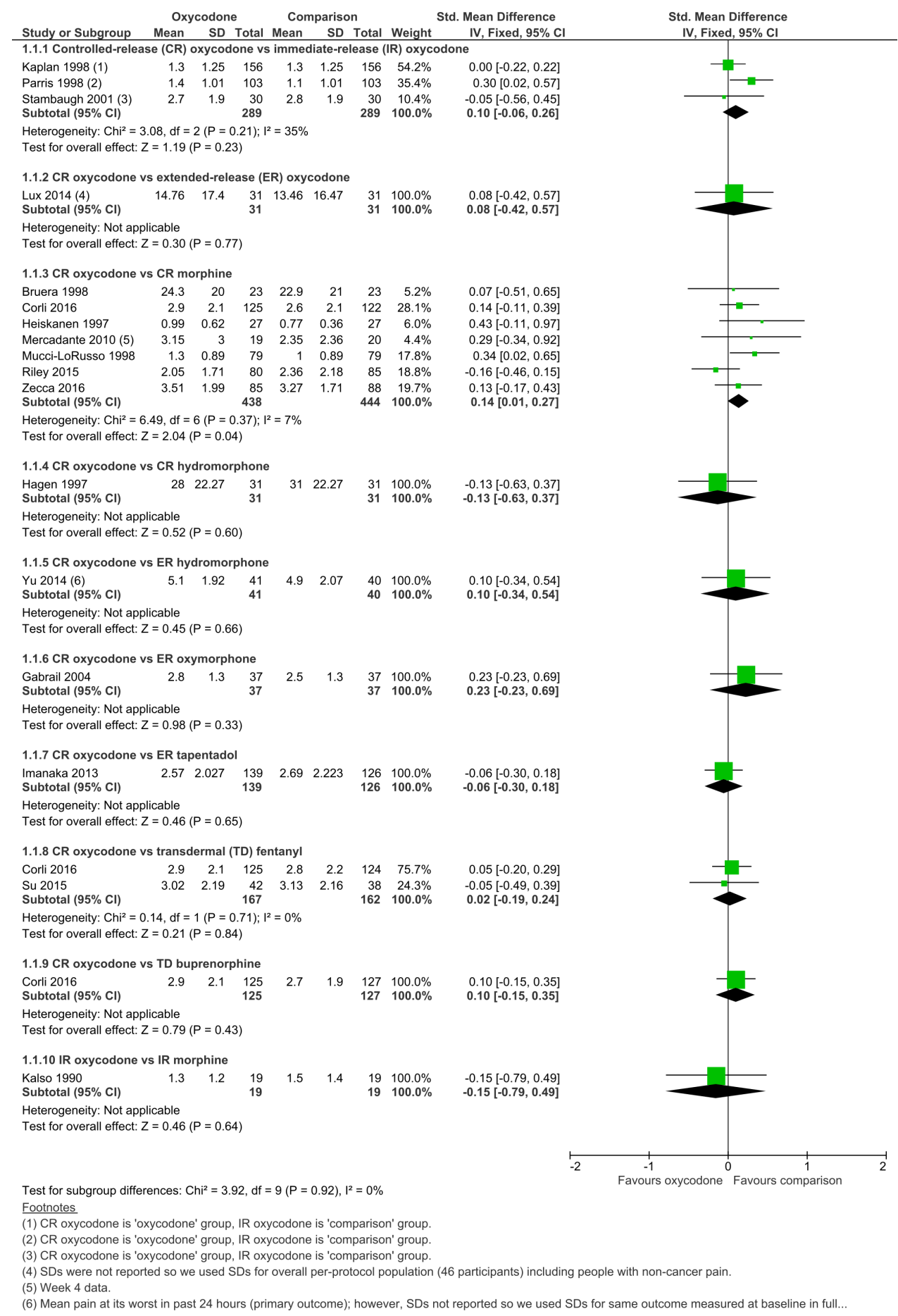

Figure 2 Mean (and SD) pain intensity/relief scores after treatment with oxycodone or its comparator, subgrouped by treatment comparisons (on the left side). Please note that pain intensity/relief was measured on different scales in some of the studies and are therefore not comparative between the studies. The meta-analysis therefore uses the standardised mean difference as the outcome measure (on the right side).

experiencing at least moderate pain relief) of the three groups did not differ significantly (CR oxycodone 53/57 participants; CR morphine 51/57 participants; CR MS Contin 52/57 participants; $\mathrm{P}=0.62) .{ }^{42}$ We judged the quality of evidence for this outcome to be low. We downgraded the quality of evidence by two levels for very serious limitations to study quality due to risk of bias (arising from under-reporting, performance/detection bias and attrition bias).

Pooled analysis showed that the pain intensity scores after treatment with CR oxycodone and TD fentanyl did not differ significantly (SMD 0.02, 95\% CI -0.19 to $0.24, \mathrm{I}^{2}=0 \%$ ). We judged the quality of evidence for this outcome to be very low. We downgraded the quality 
of evidence by two levels for very serious limitations to study quality due to risk of bias (arising from under-reporting, performance bias and detection bias) and by one level for imprecision (arising from low participant numbers).

The remaining treatment comparisons (whether included in figure 2 or not) were only examined by one study each, and none of them found any statistically significant differences in the pain scores between the respective treatments with the exception of Leow et al who, in a small single-dose crossover study with 12 participants, found that while intravenous oxycodone was associated with faster onset of pain relief relative to rectal oxycodone, rectal oxycodone was associated with a longer duration of pain relief compared with intravenous oxycodone. ${ }^{28}$ We judged the quality of the evidence for these outcomes to be low or very low as we downgraded the quality of the evidence by one or two levels for serious/very serious limitations to study quality due to risk of bias (arising from under-reporting, performance bias, detection bias and/or attrition bias) and by one or two levels for imprecision (arising from low/very low participant numbers).

\section{Adverse events}

Table 1 lists the pooled analyses of the adverse events.

Pooled analyses showed no significant difference between treatment with CR and IR oxycodone for any of the adverse events. However, the $\mathrm{I}^{2}$ statistic was $61 \%$ for headache and $55 \%$ for sweating so the results reported in table 1 for these two adverse events should be disregarded and are instead presented in figures 3 and 4. Parris et al analysed 109 participants for safety evaluation; however, it is unclear which group had 55 and which had 54 participants. ${ }^{36}$ In the meta-analyses of adverse events, we allocated 54 participants to the $\mathrm{CR}$ oxycodone group and 55 to the IR oxycodone group. Sensitivity analyses showed that allocating 55 participants to the CR oxycodone group and 54 participants to the IR oxycodone group made no difference to the conclusions. Stambaugh et al in a crossover study stated that: 'The study showed similar incidences and numbers of reports of individual adverse events considered related to the IR and CR drug' (p. 505) but did not report any formal statistical comparisons of the adverse event rates between the study groups. ${ }^{30}$ We judged the quality of evidence for adverse events to be very low for all adverse events. We downgraded the quality of evidence by two levels for very serious limitations to study quality due to risk of bias (arising from under-reporting, performance/detection bias, inadequate titration, attrition bias or a combination of these), and we downgraded the quality of the evidence by one or two levels due to imprecision (arising from low or very low event rates).

Pooled analyses of adverse events showed no significant difference between treatment with CR oxycodone and CR morphine for any of the adverse events apart from hallucinations where the RR was significantly lower after treatment with CR oxycodone than after CR morphine. Moreover, the $\mathrm{I}^{2}$ statistic was $53 \%$ for 'any adverse events' so the results reported in table 1 for this outcome should be disregarded and are instead presented in figure 5 . Zhang et al compared CR oxycodone to both CR morphine and CR MS Contin. ${ }^{42}$ In the meta-analyses of adverse events, we included CR morphine as the comparison group. Sensitivity analyses substituting the CR morphine data with the CR MS Contin data showed that whether the comparison group was CR morphine or CR MS Contin made no difference to the conclusions.

Bruera et al reported that: 'There were no statistically significant differences by treatment in mean severity for any of the elicited adverse events or in the frequency of reporting of unelicited events' (p. 3225) but presented only data on sedation and nausea VAS ratings. ${ }^{18}$ Corli et al found no significant differences between the two treatment groups in the incidences of gastralgia and breathlessness, whether they were 'any degree' or 'severe'. Severe but not 'any degree' muscle spasm myoclonus occurred significantly more often in the CR morphine group than in the CR oxycodone group. ${ }^{31}$ Heiskanen et al in a crossover trial found that vomiting was significantly more common during morphine treatment, while constipation was significantly more common during oxycodone treatment and reported no other significant differences in adverse event rates between the drugs. ${ }^{23}{ }^{24}$ Mercadante et al found no significant differences in the reported adverse events between the groups. ${ }^{34}$ Lauretti et al found that CR and IR morphine were associated with more nausea and vomiting compared with the combination of CR oxycodone and IR morphine. ${ }^{27}$ We judged the quality of evidence for this outcome to be very low for all adverse events. We downgraded the quality of evidence by two levels for very serious limitations to study quality due to risk of bias (arising from under-reporting, performance/detection bias, attrition bias or a combination of these), and we downgraded the quality of the evidence by one or two levels due to imprecision (arising from low or very low event rates).

Pooled analysis showed that there was no significant difference between CR oxycodone and TD fentanyl for dysuria and that the $\mathrm{I}^{2}$ statistic was $83 \%$ for constipation. The results reported for constipation in table 1 should therefore be disregarded and considered on a study-by-study basis only for this treatment comparison (see also figure 6). Corli et al found no significant differences between the two treatment groups in the incidences of the following adverse events, whether they were 'any degree' or 'severe': drowsiness, confusion, nausea, vomiting, constipation, dry mouth, hallucinations, gastralgia, muscle spasm myoclonus, breathlessness and itching. ${ }^{31}$ 
Table 1 List of the meta-analyses of the adverse events data from the following comparisons: CR oxycodone versus IR oxycodone, CR oxycodone versus CR morphine and CR oxycodone versus TD fentanyl

\begin{tabular}{|c|c|c|c|c|c|}
\hline Outcome and subgroup & Studies & CR OXY events/total (\%) & Comparison events/total (\%) & Risk ratio $(95 \% \mathrm{Cl})$ & $I^{2}(\%)$ \\
\hline \multicolumn{6}{|l|}{ Any adverse event } \\
\hline CR OXY versus $C R$ MOR & 3 & $142 / 196(72.4)$ & $152 / 203(74.9)$ & 0.97 (0.87 to 1.09$)$ & 53 \\
\hline \multicolumn{6}{|l|}{ Asthenia } \\
\hline CR OXY versus IR OXY & 2 & $5 / 102(4.9)$ & 9/106 (8.5) & $0.58(0.2$ to 1.68$)$ & 30 \\
\hline \multicolumn{6}{|l|}{ Confusion } \\
\hline CR OXY versus IR OXY & 2 & $3 / 78(3.8)$ & $4 / 79(5.1)$ & 0.78 (0.2 to 3.02$)$ & 25 \\
\hline CR OXY versus $C R M O R$ & 3 & $74 / 295(25.1)$ & $73 / 289(25.3)$ & 1.01 (0.78 to 1.31$)$ & 16 \\
\hline \multicolumn{6}{|l|}{ Constipation } \\
\hline CR OXY versus IR OXY & 3 & $25 / 156(16)$ & $36 / 161(22.4)$ & 0.71 (0.45 to 1.13$)$ & 38 \\
\hline CR OXY versus $C R$ MOR & 5 & $139 / 400(34.8)$ & 141/397 (35.5) & $0.98(0.82$ to 1.16$)$ & 33 \\
\hline CR OXY versus TD FEN & 2 & $88 / 171(51.5)$ & $80 / 165(48.5)$ & 1.07 (0.88 to 1.32$)$ & 83 \\
\hline \multicolumn{6}{|l|}{ Dizziness/lightheadedness } \\
\hline CR OXY versus IR OXY & 3 & 15/156 (9.6) & $21 / 161(13)$ & 0.74 (0.4 to 1.37$)$ & 15 \\
\hline CR OXY versus CR MOR & 3 & 9/186 (4.8) & 12/181 (6.6) & 0.76 (0.33 to 1.76$)$ & 0 \\
\hline \multicolumn{6}{|l|}{ Drowsiness/somnolence } \\
\hline CR OXY versus IR OXY & 3 & $36 / 156(23.1)$ & $36 / 161(22.4)$ & 1.03 (0.69 to 1.54$)$ & 0 \\
\hline CR OXY versus CR MOR & 5 & $121 / 400(30.3)$ & 134/398 (33.7) & 0.9 (0.75 to 1.08$)$ & 0 \\
\hline \multicolumn{6}{|l|}{ Dry mouth } \\
\hline CR OXY versus IR OXY & 3 & 10/156 (6.4) & 9/161 (5.6) & $1.14(0.48$ to 2.75$)$ & 0 \\
\hline CR OXY versus CR MOR & 4 & $89 / 343(25.9)$ & $89 / 341(26.1)$ & 1.01 (0.8 to 1.26$)$ & 32 \\
\hline \multicolumn{6}{|l|}{ Dysuria } \\
\hline CR OXY versus $C R$ MOR & 2 & $17 / 186(9.1)$ & 24/186 (12.9) & $0.71(0.4$ to 1.26$)$ & 0 \\
\hline CR OXY versus TD FEN & 2 & 19/171 (11.1) & $16 / 165(9.7)$ & 1.15 (0.62 to 2.16$)$ & 0 \\
\hline \multicolumn{6}{|l|}{ Hallucinations } \\
\hline CR OXY versus $C R$ MOR & 4 & $14 / 349(4)$ & $27 / 347(7.8)$ & 0.52 (0.28 to 0.97$)$ & 0 \\
\hline \multicolumn{6}{|l|}{ Headache } \\
\hline CR OXY versus IR OXY & 3 & $8 / 156(5.1)$ & 10/161 (6.2) & 0.83 (0.34 to 2.02$)$ & 61 \\
\hline \multicolumn{6}{|l|}{ Insomnia } \\
\hline CR OXY versus IR OXY & 2 & $5 / 132(3.8)$ & $5 / 137(3.6)$ & 1.04 (0.31 to 3.53$)$ & 35 \\
\hline \multicolumn{6}{|l|}{ Nausea } \\
\hline CR OXY versus IR OXY & 3 & $32 / 156(20.5)$ & $39 / 161(24.2)$ & 0.85 (0.56 to 1.28$)$ & 0 \\
\hline CR OXY versus CR MOR & 5 & $108 / 400(27)$ & 106/398 (26.6) & 1.02 (0.82 to 1.26$)$ & 0 \\
\hline \multicolumn{6}{|l|}{ Nervousness } \\
\hline CR OXY versus IR OXY & 2 & $5 / 102(4.9)$ & 9/106 (8.5) & $0.57(0.2$ to 1.64$)$ & 0 \\
\hline \multicolumn{6}{|l|}{ Pruritus } \\
\hline CR OXY versus IR OXY & 3 & $13 / 156(8.3)$ & 9/161 (5.6) & 1.46 (0.65 to 3.25$)$ & 33 \\
\hline$C R O X Y$ versus $C R M O R$ & 4 & $29 / 343(8.5)$ & 36/341 (10.6) & 0.81 (0.51 to 1.29$)$ & 0 \\
\hline \multicolumn{6}{|l|}{ Sweating } \\
\hline CR OXY versus IR OXY & 2 & $5 / 132(3.8)$ & $8 / 137(5.8)$ & 0.65 (0.22 to 1.93$)$ & 55 \\
\hline \multicolumn{6}{|l|}{ Vomiting } \\
\hline CR OXY versus IR OXY & 3 & $18 / 156(11.5)$ & 28/161 (17.4) & $0.66(0.38$ to 1.15$)$ & 18 \\
\hline CR OXY versus CR MOR & 5 & $60 / 400(15)$ & $64 / 398(16.1)$ & $0.94(0.68$ to 1.29$)$ & 2 \\
\hline \multicolumn{6}{|c|}{ Discontinuation due to adverse events } \\
\hline CR OXY versus IR OXY & 3 & $11 / 156(7.1)$ & 19/161 (11.8) & $0.6(0.29$ to 1.22$)$ & 0 \\
\hline CR OXY versus $C R M O R$ & 3 & 9/196 (4.6) & $9 / 203(4.4)$ & $1.06(0.43$ to 2.6$)$ & 48 \\
\hline
\end{tabular}

CR, controlled release; FEN, fentanyl; IR, immediate release; MOR, morphine; OXY, oxycodone; TD, transdermal.

Su et $^{4 l^{40}}$ reported that the rates of nausea and vomiting $(\mathrm{P}=0.026)$ and constipation $(\mathrm{P}=0.022)$ were significantly higher in the CR oxycodone group than in the TD fentanyl group, and that the rates of dizziness and lethargy did not differ significantly between the groups
(Ps $>0.65$ ). We judged the quality of evidence for this outcome to be very low. We downgraded the quality of evidence by two levels for very serious limitations to study quality due to risk of bias (arising from under-reporting, performance bias and detection bias) and by 


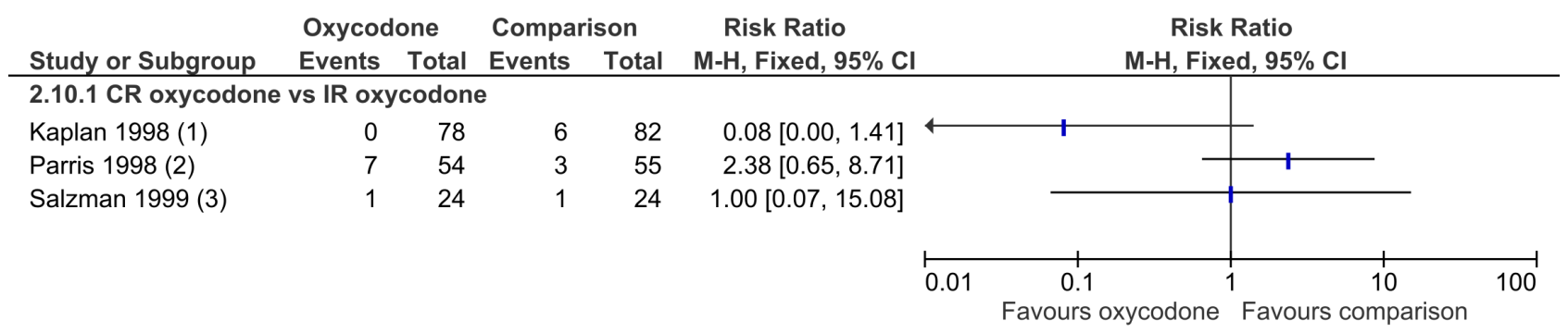

$\underline{\text { Footnotes }}$

(1) Please note that $C R$ oxycodone is input as the 'oxycodone' group and IR oxycodone is input as the 'comparison' group.

(2) Please note that CR oxycodone is input as the 'oxycodone' group and IR oxycodone is input as the 'comparison' group.

(3) Please note that CR oxycodone is input as the 'oxycodone' group and IR oxycodone is input as the 'comparison' group.

Figure 3 Headache (adverse events) rates in patients receiving treatment with controlled-release (CR) and immediate-release (IR) oxycodone.

one or two levels (depending on the event rates of the individual adverse events) for imprecision (arising from low or very low event rates).

A number of the remaining studies did not report adverse events split or inferentially analysed by treatment group. ${ }^{12} 161720212832$ Of those studies that did perform the analyses, ${ }^{13} 22252631$ no significant differences were found between the treatment groups with the following exceptions:

- Drowsiness occurred more frequently during treatment with CR oxycodone compared with $\mathrm{CR}$ hydromorphone. ${ }^{22}$

- 'Any degree', but not severe, breathlessness occurred more often in the TD buprenorphine group than in the CR oxycodone group. ${ }^{31}$

- Nausea was significantly more common with oral morphine treatment compared with intravenous oxycodone, IR oxycodone and intravenois morphine. ${ }^{25} 26$

We judged the quality of evidence for these outcomes to be very low to moderate. We downgraded the quality of evidence by none to two levels for no, serious or very serious limitations to study quality due to risk of bias (arising from under-reporting, performance bias, detection bias and/or attrition bias) and one or two levels for imprecision (arising from low or very low participant numbers/event rates).

See also the results section of the online supplementary file for tables of all the adverse events reported by the included studies along with the remaining forest plots of the pooled analyses reported in table 1 .

\section{Quality of life}

The majority of the studies did not report quality of life, ${ }^{12} 13$ 16-19 22252628313234 37-39 41 42 but Mucci-LoRusso et al reported no clinically significant changes in quality of life for either CR oxycodone or CR morphine group but did not show results or analyses, ${ }^{35}$ Gabrail et al observed no differences in quality of life (general activity, mood, walking ability, normal work, relationships with others, sleep and enjoyment of life) between CR oxycodone to ER oxymorphone $e^{20} 21$ and $\mathrm{Su} e \mathrm{al}^{40}$ found no differences in quality of life between CR oxycodone and TD fentanyl as measured by the Karnofsky Performance Status. Moreover, treatment acceptability ratings were not significantly different between CR and IR oxycodone, ${ }^{29} 303336$ or CR oxycodone and CR morphine, ${ }^{27} 35$ although Heiskanen et al found that the mean daily acceptability of treatment ratings were significantly higher for CR morphine than for CR oxycodone. ${ }^{2324}$

We judged the quality of evidence for this outcome to be low to very low. We downgraded the quality of evidence by two levels for very serious limitations to study quality due to risk of bias (arising from under-reporting and attrition bias), and we downgraded none,

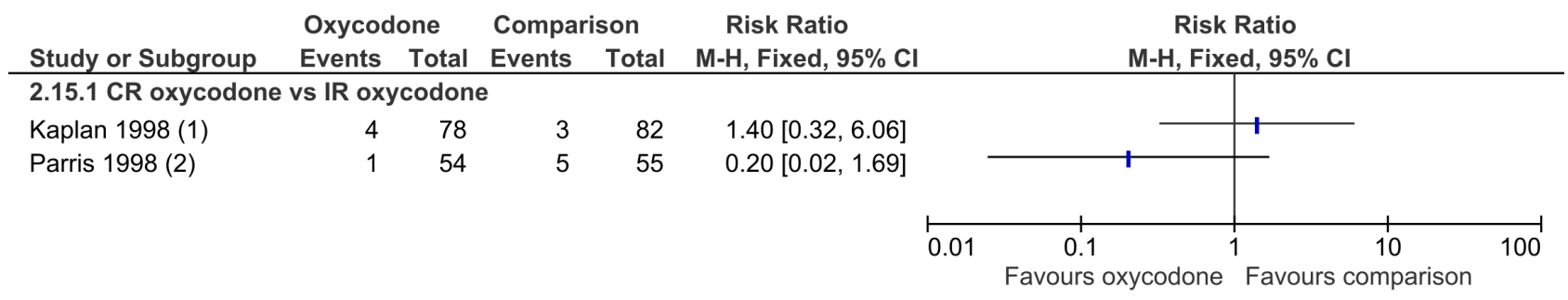

Footnotes

(1) Please note that CR oxycodone is input as the 'oxycodone' group and IR oxycodone is input as the 'comparison' group.

(2) Please note that CR oxycodone is input as the 'oxycodone' group and IR oxycodone is input as the 'comparison' group.

Figure 4 Sweating (adverse event) rates in patients receiving treatment with controlled-release (CR) and immediate-release (IR) oxycodone. 


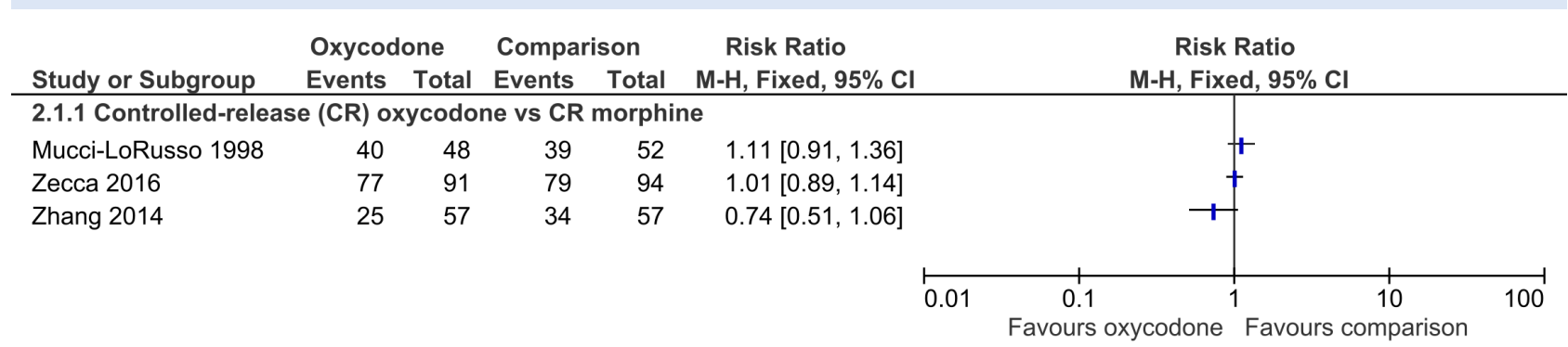

Figure 5 'Any adverse event' rates in patients receiving treatment with CR oxycodone and CR morphine.

one or two levels for imprecision (arising from low or very low participant numbers).

\section{Patient preference}

Treatment preference did not differ significantly between CR oxycodone and CR morphine, ${ }^{18} 19$ CR oxycodone and CR hydromorphone, ${ }^{22}$ or between intravenous oxycodone followed by IR oxycodone and intravenous morphine followed by IR morphine. ${ }^{25} 26$ Gabrail et al reported no data for participant preference but found that $78.3 \%$ of participants rated oxycodone as 'excellent,' 'very good' or 'good' with
$86.4 \%$ of the participants giving oxymorphone such ratings. ${ }^{20} 21$

We judged the quality of evidence for this outcome to be very low. We downgraded the quality of evidence by two levels for very serious limitations to study quality due to risk of bias (arising from under-reporting and attrition bias), and we downgraded the quality of the evidence by two levels due to imprecision (arising from very low participant numbers).

Patient preference was not reported by the remaining studies. ${ }^{12} 131617232427-40$ See also the

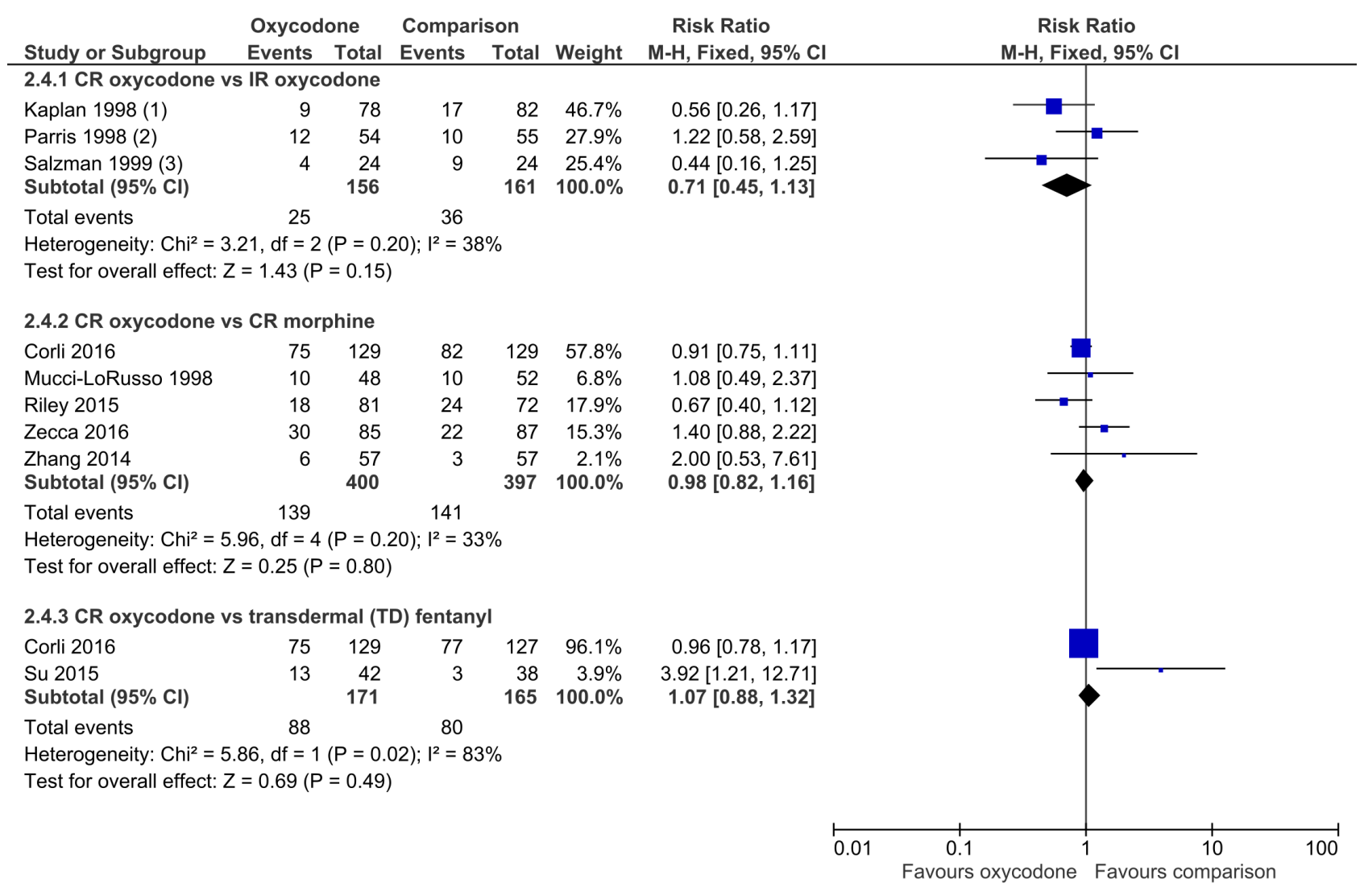

Footnotes

(1) Please note that CR oxycodone is input as the 'oxycodone' group and IR oxycodone is input as the 'comparison' group.

(2) Please note that CR oxycodone is input as the 'oxycodone' group and IR oxycodone is input as the 'comparison' group.

(3) Please note that CR oxycodone is input as the 'oxycodone' group and IR oxycodone is input as the 'comparison' group.

Figure 6 Constipation (adverse event) rates in patients receiving treatment with controlled-release (CR) and immediate-release (IR) oxycodone, CR oxycodone and CR morphine and CR morphine and transdermal (TD) fentanyl, respectively. Please note: for the CR morphine and TD fentanyl comparison, the subtotal listed should be disregarded due to substantial heterogeneity $\left(I^{2}=83 \%\right)$, instead the included data should be considered on a study-by-study basis only. 
'Summary of findings' tables in the results section of the online supplementary file.

\section{DISCUSSION}

There was no difference in pain intensity or adverse event rates after treatment with either CR or IR oxycodone. Three of the four studies also found no difference in treatment acceptability between the comparisons. We noted that IR oxycodone was given every 6 hours rather than every 4 hours in these studies. This might have biased the efficacy data in favour of CR oxycodone; however, the adverse effect data suggest that giving IR oxycodone every 4 hours (more frequently) would have resulted in greater adverse effects, which would have mitigated advantages in efficacy.

Nine studies compared CR oxycodone with CR morphine, and pooled analysis of seven of these nine studies suggested that pain intensity did differ significantly between the treatments. However, this result was not corroborated by a sensitivity analysis that excluded the two crossover trials included in the overall analysis. There were no marked differences in terms of treatment acceptability or quality of life ratings observed between the treatments. Zhang et al also found no significant differences in pain relief rates between CR oxycodone, CR morphine and CR MS Contin. ${ }^{42}$ Pooled analyses of the adverse event rates also found no differences between the treatments for any of the adverse events, with the exception of hallucinations, which participants treated with CR morphine were at almost double the risk of experiencing compared with participants treated with CR oxycodone. However, these findings contrast somewhat with those reported by Lauretti et $a l,{ }^{27}$ which concluded that CR oxycodone combined with IR morphine is associated with superior analgesia and lower or similar rates of adverse events than a combination of CR and IR morphine. ${ }^{27}$

Two studies compared CR oxycodone with TD fentanyl and pooled analysis of them found that the pain intensity scores after treatment with CR oxycodone and TD fentanyl did not differ significantly. One of the studies also found that quality of life did not differ between the treatments, but there was some disagreement between the study results in terms of adverse events with one of the studies finding that the rates of nausea and vomiting, and constipation were significantly higher in the CR oxycodone group than in the TD fentanyl group, whereas the other study reported no treatment group differences in (these and other) adverse event rates.

The remaining studies all compared either oxycodone in different formulations or oxycodone with different alternative opioids and none of them found any clear superiority or inferiority of oxycodone for cancer pain, neither as an analgesic agent nor in terms of adverse event rates or treatment acceptability.

Although the findings of this review are applicable to the population and comparisons defined for this review, that is, adults with cancer who need treatment with strong opioids for cancer pain, they should be taken in the context that this review found 23 studies that were eligible for inclusion and these studies reported on 13 different comparisons involving oxycodone and included only 2648 participants. Moreover, for some of the outcomes (participant preference and quality of life), there were extremely few data available. To somewhat mitigate this shortfall, we reported treatment acceptability as a proxy. However, that does not change the fact that the evidence base for the effectiveness and tolerability of oxycodone (relative or absolute) for pain in adults with cancer was very limited, and it did not allow us to examine the effectiveness and tolerability of oxycodone in detail through participant or treatment subgroup analyses. The current evidence base would therefore benefit from more well-designed, large RCTs.

The quality of the evidence for all the outcomes was low or very low, meaning we have little confidence in the effect estimate and the true effect may be substantially different from the estimate of the effect. This is due to imprecision (low participant numbers) in some cases and serious or very serious study limitations in all cases. In general, the assessment of the quality of the included studies was limited by a great extent of under-reporting in the studies, especially for the participant selection items (random sequence generation and allocation concealment), while blinding appeared to be reasonably well undertaken overall, both in terms of treatment performance and outcome assessment. However, as is not unusual for pain research, the results were substantially compromised by attrition, with data missing from just under $20 \%$ of the enrolled/randomised participants for efficacy and from more than $10 \%$ for safety. These are substantial proportions, and while it did not appear to be selective attrition, the results must be interpreted with caution.

Our conclusions are in agreement with those of other systematic reviews in this area despite some differences in inclusion criteria and analysis strategy. ${ }^{43-46}$

\section{CONCLUSIONS}

\section{Implications for practice}

We found low-quality evidence that oxycodone offers similar levels of cancer pain relief and adverse events to other strong opioids including morphine, which is commonly considered the gold standard strong opioid. Although we identified a clinically insignificant benefit on pain relief in favour of CR morphine compared with CR oxycodone, this did not persist following sensitivity analysis, so we do not consider this important. We found an increased frequency of hallucinations after treatment with CR morphine $(7.8 \%)$ compared with CR oxycodone (4\%), but we also found a numerically higher frequency of myoclonus (another excitatory opioid adverse effect) with CR oxycodone, and we did not find any differences in reported drowsiness or confusion. The interpretation 
of an increased relative risk of hallucinations should therefore be treated with caution given the low quality of evidence. Our findings are consistent with current international guidance that oxycodone can be used first line as an alternative to morphine. However, because it is cheaper, morphine generally remains the first-line opioid of choice for adults with cancer-related pain.

\section{Implications for research}

We found that the current evidence base is composed of studies that contained small numbers of participants in which there was a significant $(19 \%)$ dropout rate. For example, the direct comparison meta-analysis between oxycodone and morphine was based on fewer than 450 cancer participants in each treatment group; this was a very small evidence base. However, given the absence of important differences within this analysis, it seems unlikely that larger head-to-head studies of oxycodone versus morphine will be justified. For future cancer pain studies, developing a single outcome that combines good pain control (no more than mild on a verbal rating scale) with acceptable adverse effects (perhaps no more than mild severity on any adverse event) would enable a clearer comparison between any analgesics used in this context.

Acknowledgements We would like to thank Yuhong Yuan (Department of Medicine, Division of Gastroenterology, McMaster University, Canada) and Yu-Tian Xiao (School of Clinical Medicine, Fudan University, China) for their help with the translation of Chinese papers, and Joanne Abbott at the Cochrane Pain, Palliative and Supportive Care Review Group $(\mathrm{PaPaS})$ for her assistance in devising and executing the search strategy. We would also like to thank the Cochrane Pain Palliative and Supportive Care Review group for all their assistance in the publication of the original and updated Cochrane review that this article is an abridged version of. Cochrane Review Group funding acknowledgement: this project was supported by the National Institute for Health Research (NIHR), via Cochrane Infrastructure funding to Cochrane PaPaS.

Contributors MS-H and MIB conceived and designed the review and wrote the protocol. SA devised and undertook the search strategy. MS-H, NB and JSH screened the search results and performed the data extraction and 'risk of bias' assessment of the included studies. MS-H devised and performed the analysis strategy and wrote the first draft of the full review. MIB interpreted the results and wrote the 'Authors conclusions' section. All the authors approved the final version of the review.

Disclaimer The views and opinions expressed therein are those of the authors and do not necessarily reflect those of the Systematic Reviews Programme, NIHR, National Health Service, or the Department of Health.

Competing interests $\mathrm{MIB}$ is a specialist palliative medicine physician and manages people with cancer pain. MIB received lecture fees from Pfizer in 2016.

Provenance and peer review Not commissioned; externally peer reviewed.

(C) Article author(s) (or their employer(s) unless otherwise stated in the text of the article) 2018. All rights reserved. No commercial use is permitted unless otherwise expressly granted.

\section{REFERENCES}

1 Breivik H, Cherny N, Collett B, et al. Cancer-related pain: a pan-European survey of prevalence, treatment, and patient attitudes. Ann Oncol 2009;20:1420-33.

2 Greco MT, Roberto A, Corli O, et al. Quality of cancer pain management: an update of a systematic review of undertreatment of patients with cancer. J Clin Oncol 2014;32:4149-54.

3 Ziegler L, Mulvey M, Blenkinsopp A, et al. Opioid prescribing for patients with cancer in the last year of life: a longitudinal population cohort study. Pain 2016;157:2445-51.

4 Gudin J. Opioid therapies and cytochrome p450 interactions. J Pain Symptom Manage 2012;44:S4-S14.

5 Leppert W. Role of oxycodone and oxycodone/naloxone in cancer pain management. Pharmacol Rep 2010;62:578-91.

6 World Health Organization (WHO). Cancer pain relief. Geneva: World Health Organization (WHO), 1986.

7 Bennett MI. What evidence do we have that the WHO analgesic ladder is effective in cancer pain? In: McQuay HJ, Moore R, Kalso E, eds. Systematic reviews in pain research; methodology refined. Seattle (WA): IASP Press, 2008:303-13.

8 Ventafridda V, Tamburini M, Caraceni A, et al. A validation study of the WHO method for cancer pain relief. Cancer 1987;59:850-6.

9 Caraceni A, Hanks G, Kaasa S, et al. Use of opioid analgesics in the treatment of cancer pain: evidence-based recommendations from the EAPC. Lancet Oncol 2012;13:e58-e68.

10 Schmidt-Hansen M, Bennett MI, Arnold S, et al. Oxycodone for cancer-related pain. Cochrane Database Syst Rev 2017;8:CD003870.

11 Higgins JPT, Green S, eds. Cochrane handbook for systematic reviews of interventions version 5.1.0 (updated March 2011): The Cochrane Collaboration, 2011. handbook.cochrane.org

12 Lux EA, Janecki M, Maritz MA. Clinical evaluation of the first oxycodone once daily prolonged release tablet in moderate to severe chronic pain: a randomized, double-blind, multicenter, cross-over, non-inferiority study to investigate efficacy and safety in comparison with an established oxycodone twice daily prolonged release tablet. Curr Med Res Opin 2014;30:2365-75.

13 Yu S, Shen W, Yu L, et al. Safety and efficacy of oncedaily hydromorphone extended-release versus twice-daily oxycodone hydrochloride controlled-release in chinese patients with cancer pain: a phase 3 , randomized, double-blind, multicenter study. J Pain 2014;15:835-44.

14 Review manager (RevMan) [Computer program]. version 5.3. Copenhagen: The Nordic Cochrane Centre, The Cochrane Collaboration, 2014.

15 GRADEpro guideline development tool [Software] [Computer program]: McMaster University, 2015 (developed by Evidence Prime, Inc.), 2015. www.gradepro.org

16 Beaver WT, Wallenstein SL, Rogers A, et al. Analgesic studies of codeine and oxycodone in patients with cancer. I. Comparisons of oral with intramuscular codeine and of oral with intramuscular oxycodone. J Pharmacol Exp Ther 1978;207:92-100.

17 Beaver WT, Wallenstein SL, Rogers A, et al. Analgesic studies of codeine and oxycodone in patients with cancer. II. Comparisons of intramuscular oxycodone with intramuscular morphine and codeine. J Pharmacol Exp Ther 1978;207:101-8.

18 Bruera E, Belzile M, Pituskin E, et al. Randomized, doubleblind, cross-over trial comparing safety and efficacy of oral controlled-release oxycodone with controlled-release morphine in patients with cancer pain. J Clin Oncol 1998;16:3222-9.

19 Comerford T, Bruera E. Efficacy of controlled-release oxycodone. J Clin Oncol 1999; 17:730d.

20 Gabrail NY, Dvergsten C, Ahdieh H. Establishing the dosage equivalency of oxymorphone extended release and oxycodone 
controlled release in patients with cancer pain: a randomized controlled study. Curr Med Res Opin 2004;20:911-8.

21 Gabrail NY, Dvergsten C, Ma T, et al. Oxymorphone extended-release (ER) provides safe, effective, and rapid analgesia during opioid radiation: results of a randomized, double-blind, crossover, comparative study with oxycodone controlled-release (CR) [abstract]. Proc Am Society Clin Oncol 2003.

22 Hagen NA, Babul N. Comparative clinical efficacy and safety of a novel controlled-release oxycodone formulation and controlled-release hydromorphone in the treatment of cancer pain. Cancer 1997;79:1428-37.

23 Heiskanen T, Kalso E. Controlled-release oxycodone and morphine in cancer related pain. Pain 1997;73:37-45.

24 Heiskanen TE, Ruismäki PM, Seppälä TA, et al. Morphine or oxycodone in cancer pain? Acta Oncol 2000;39:941-7.

25 Kalso E, Vainio A, Mattila MJ, et al. Morphine and oxycodone in the management of cancer pain: plasma levels determined by chemical and radioreceptor assays. Pharmacol Toxicol 1990;67:322-8.

26 Kalso E, Vainio A. Morphine and oxycodone hydrochloride in the management of cancer pain. Clin Pharmacol Ther 1990;47:639-46.

27 Lauretti GR, Oliveira GM, Pereira NL. Comparison of sustained-release morphine with sustained-release oxycodone in advanced cancer patients. Br J Cancer 2003;89:2027-30.

28 Leow KP, Cramond T, Smith MT. Pharmacokinetics and pharmacodynamics of oxycodone when given intravenously and rectally to adult patients with cancer pain. Anesth Analg 1995;80:296-302.

29 Stambaugh JE, Reder RF, Stambaugh M. Doubleblind, randomized, two-period crossover efficacy and pharmacokinetic comparison of immediate-release oxycodone (IR) and controlled-release oxycodone (CR) in cancer patients with pain. Clin Pharmacol Ther 1997;61:197.

30 Stambaugh JE, Reder RF, Stambaugh MD, et al. Double-blind, randomized comparison of the analgesic and pharmacokinetic profiles of controlled- and immediate-release oral oxycodone in cancer pain patients. J Clin Pharmacol 2001;41:500-6.

31 Corli O, Floriani I, Roberto A, et al. Are strong opioids equally effective and safe in the treatment of chronic cancer pain? A multicenter randomized phase IV 'real life' trial on the variability of response to opioids. Ann Oncol 2016;27:1107-15.

32 Imanaka K, Tominaga Y, Etropolski M, et al. Efficacy and safety of oral tapentadol extended release in Japanese and Korean patients with moderate to severe, chronic malignant tumor-related pain. Curr Med Res Opin 2013;29:1399-409.

33 Kaplan R, Parris WC, Citron ML, et al. Comparison of controlled-release and immediate-release oxycodone tablets in patients with cancer pain. J Clin Oncol 1998;16:3230-7.
34 Mercadante S, Tirelli W, David F, et al. Morphine versus oxycodone in pancreatic cancer pain: a randomized controlled study. Clin J Pain 2010;26:794-7.

35 Mucci-LoRusso P, Berman BS, Silberstein PT, et al. Controlled-release oxycodone compared with controlledrelease morphine in the treatment of cancer pain: a randomized, double-blind, parallel-group study. Eur J Pain 1998;2:239-49.

36 Parris WC, Johnson BW, Croghan MK, et al. The use of controlled-release oxycodone for the treatment of chronic cancer pain: a randomized, double-blind study. J Pain Symptom Manage 1998;16:205-11.

37 Riley J, Branford R, Droney J, et al. Morphine or oxycodone for cancer-related pain? A randomized, open-label, controlled trial. J Pain Symptom Manage 2015;49:161-72.

38 Riley J, Branford R, Droney J, et al. A randomised controlled trial of oral morphine versus oral oxycodone for the treatment of pain in cancer patients. Palliat Med 2012;26:386.

39 Salzman RT, Roberts MS, Wild J, et al. Can a controlledrelease oral dose form of oxycodone be used as readily as an immediate-release form for the purpose of titrating to stable pain control? J Pain Symptom Manage 1999;18:271-9.

$40 \mathrm{Su}$ J, Zhu Y, Wu W, et al. Observation of curative effects and adverse effects of oxycodone hydrochloride controlled-release tablets and fentanyl transdermal patches on the treatment of moderate or severe cancer pain. Anti-Tumor Pharmacy 2015;5:444-8.

41 Zecca E, Brunelli C, Bracchi P, et al. Comparison of the tolerability profile of controlled-release oral morphine and oxycodone for cancer pain treatment. An open-label randomized controlled trial. J Pain Symptom Manage 2016;52:783-94.

42 Zhang WZ, Yu WJ, Zhao XL, et al. Pharmacoeconomics evaluation of morphine, MS contin and oxycodone in the treatment of cancer pain. Asian Pac J Cancer Prev 2014; 15:8797-800.

43 Bekkering GE, Soares-Weiser K, Reid K, et al. Can morphine still be considered to be the standard for treating chronic pain? A systematic review including pair-wise and network metaanalyses. Curr Med Res Opin 2011;27:1477-91.

44 Caraceni A, Pigni A, Brunelli C. Is oral morphine still the first choice opioid for moderate to severe cancer pain? A systematic review within the European Palliative Care Research Collaborative guidelines project. Palliat Med 2011;25:402-9.

45 King SJ, Reid C, Forbes K, et al. A systematic review of oxycodone in the management of cancer pain. Palliat Med 2011;25:454-70.

46 Reid CM, Martin RM, Sterne JA, et al. Oxycodone for cancerrelated pain: meta-analysis of randomized controlled trials. Arch Intern Med 2006;166:837-43. 